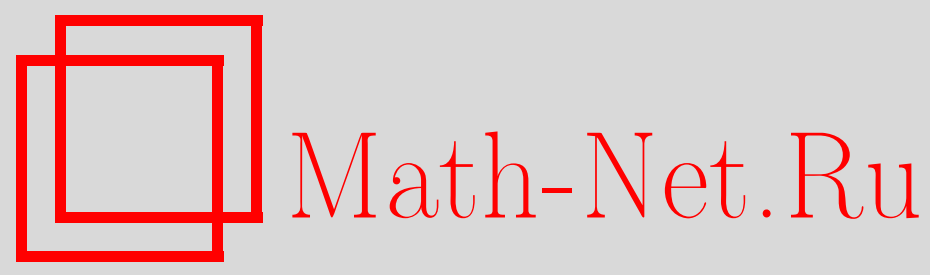

И. В. Протасов, Малые системы образующих групп, Матем. заметки, 2004, том 76, выпуск 3, 420-426

DOI: https://doi.org/10.4213/mzm118

Использование Общероссийского математического портала Math-Net.Ru подразумевает, что вы прочитали и согласны с пользовательским соглашением http://www .mathnet.ru/rus/agreement

Параметры загрузки:

IP : 3.85 .7 .115

26 апреля 2023 г., 18:03:04

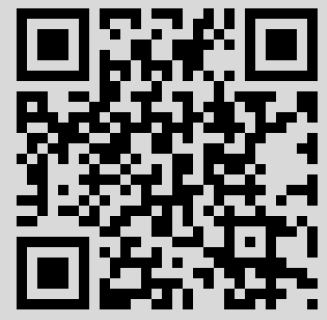


УДК 512.536

\section{МАЛЫЕ СИСТЕМЫ ОБРАЗУЮЩИХ ГРУПП}

\section{И.В. Протасов}

Подмножество $S$ группы $G$ называется большим (большим слева), если найдется такое конечное подмножество $K$, что $G=K S=S K(G=K S)$. Подмножество $S$ группы $G$ назьвается малым (мальм слева), если подмножество $G \backslash K S K(G \backslash K S)$ большое (большое слева). Доказаны следующие утверждения:

1) любая бесконечная группа порождается некоторым малым подмножеством;

2 ) в любой бесконечной группе $G$ существует такое малое слева подмножество $S$, что $G=S S^{-1}$

3) любую бесконечную группу можно разбить на счетное число малых слева подмножеств, каждое из которых порождает группу.

Библиограффия: 6 названий.

Подмножество $L$ группы $G$ назьвается боль иим, если существует такое конечноеподмножество $K \subseteq G$, что $G=K L=L K$. Подмножество $S$ групшы $G$ назьвается малы.м, если подмножество $G \backslash K S K$ большое для любого конечного подмножества $K$. Семейство всех малых подмножеств образует идеал в булевой алгебре всех подмножеств группы [1].

Верно ли, что любая бесконечная группа порождается некоторым малым подмножеством?

Для абелевых групп это так [2]. Первая теорема этой заметки дает ответ на данньй вопрос. Для подмножества $A$ группы $G$ обозначим $\operatorname{gp}(A)$ подгрупшу групшы $G$, порожденную подмножеством $A$.

Теорема 1. Пусть $G$ - бесконечная группа, $A \subseteq G u G=\operatorname{gp}(A)$. Тогда существует малое подмножество $S \subseteq A$ такое, что $G=\operatorname{gp}(S)$. Eсли группа $G$ аменабельна, то подмножество $S$ можнн выбрать так, что $\mu(S)=0$ для любой банаховой меры $\mu$ на $G$.

ДокАЗАТЕЛЬСТво. Так как любое конечное подмножество бесконечной групшы мало, можно считать, что $G \neq \operatorname{gp}(F)$ для любого конечного подмножества $F \subseteq A$. Построим индуктивно систему образующих $S=\left\{x_{\alpha}: \alpha<\gamma\right\}$ групшы $G$ такую, что $S \subseteq A$ и $x_{\alpha} \notin G_{\alpha}$, где $G_{\alpha}$ - подгруппа, порожденная подмножеством $\left\{x_{\beta}: \beta<\alpha\right\}, 0<\alpha<\gamma$. Докажем, что подмножество $S$ мало. Возьмем любое конечное подмножество $K \subset G$, $|K|=k$, и покажем, что подмножество $G \backslash K S K$ большое. Выберем ординал $\beta<\gamma$ так, чтобы $K \subset G_{\beta}$ и $\left|G_{\beta}\right|>k^{2}$. Положим $X=\left\{x_{\alpha}: \alpha \geqslant \beta\right\}$. Поскольку $K x_{\alpha} K \subseteq$ $G_{\alpha+1} \backslash G_{\alpha}$, то

$$
\left|K X K \cap\left(G_{\alpha+1} \backslash G_{\alpha}\right)\right| \leqslant k^{2}
$$


для любых $\alpha \geqslant \beta$. Выберем любое конечное подмножество $F_{1} \subseteq G_{\beta},\left|F_{1}\right|>k^{2}$ и положим $F=F_{1} \cup x_{\beta} F_{1}$. Покажем, что

$$
G=F^{-1}(G \backslash K S K)=(G \backslash K S K) F^{-1} .
$$

Допустим противное и выберем элемент $g \in G$ так, что $F g \subset K S K$ либо $g F \subset K S K$. Если $g \notin G_{\beta}$, то $g \in G_{\alpha+1} \backslash G_{\alpha}$ для некоторого ординала $\alpha \geqslant \beta$. Следовательно, либо $F g \subseteq G_{\alpha+1} \backslash G_{\alpha}$, либо $g F \subseteq G_{\alpha+1} \backslash G_{\alpha}$, что противоречит (*). Если $g \in G_{\beta}$, то либо $\left(x_{\beta} F_{1}\right) g \subseteq G_{\beta+1} \backslash G_{\beta}$, либо $g\left(x_{\beta} F_{1}\right) \subseteq G_{\beta+1} \backslash G_{\beta}$, что противоречит $(*)$.

Предположим, что $G$ аменабельна и докажем, что $\mu(S)=0$. Допустим противное и выберем натуральное число $n$ так, что $\mu(S)>1 / n$. Положим $Y=\left\{x_{\alpha}: n \leqslant \alpha<\gamma\right\}$. Тогда $Y \subset S$ и подмножество $S \backslash Y$ конечно. Следовательно, $\mu(Y)=\mu(S)$. Рассмотрим семейство подмножеств $x_{0} Y, x_{1} Y, \ldots, x_{n-1} Y$. По построению $S$ это семейство дизъюнктно. Так как $\mu\left(x_{0} Y\right)=\mu\left(x_{1} Y\right)=\cdots=\mu\left(x_{n-1} Y\right)=\mu(Y)$ и $\mu(Y)>1 / n$, то $\mu(G)>1$; противоречие.

Подмножество $L$ группы $G$ называется больиим слев $a$, если $G=K L$ для некоторого конечного подмножества $K \subseteq G$. Подмножество $S$ групшы $G$ называется малылм слева, если подмножество $G \backslash K S$ большое слева для любого конечного подмножества $K \subseteq G$.

Теорема 2. В любой бесконечной группе $G$ существует малое слева подмножество $S$ такое, что $G=S S^{-1}$. Если группа $G$ аменабельна, то подмножество $S$ можно выбрать так, что $\mu(S)=0$ для любой банаховой меры $\mu$ на $G$.

ДоказАТЕЛЬСтво. Пусть $\left\{g_{\alpha}: \alpha<\gamma\right\}$ - пересчет групшы $G, g_{0}$ - единица, $G_{\beta}=$ $\left\{g_{\alpha}: \alpha \leqslant \beta\right\}, \beta<\gamma$. Так как $\left|G_{\beta}\right|<\gamma$ для любого ординала $\beta<\gamma$, легко построить индуктивно подмножество $\left\{a_{\alpha}: \alpha<\gamma\right\}$ группы $G$, удовлетворяющее условию

$$
\left(G_{\beta} a_{\alpha} \cup G_{\beta} g_{\alpha} a_{\alpha}\right) \cap\left(G_{\beta} a_{\beta} \cup G_{\beta} g_{\beta} a_{\beta}\right)=\varnothing .
$$

Положим $S=\left\{a_{\alpha}: \alpha<\gamma\right\} \cup\left\{g_{\alpha} a_{\alpha}: \alpha<\gamma\right\}$. Очевидно, $G=S S^{-1}$. Покажем, что подмножество $S$ мало слева. Зафиксируем любое конечное подмножество $F \subset G$ и выберем $\beta_{1}<\gamma$ так, что $F \subset G_{\beta_{1}}$. Возьмем любой элемент $g \in G \backslash F$ и выберем $\beta_{2} \geqslant \beta_{1}$ так, что $g \notin F g_{\alpha} \cup F g_{\alpha}^{-1}$ для всех $\alpha \geqslant \beta_{2}$. Выберем $\beta \geqslant \beta_{2}$ так, чтобы $F^{-1} g \subseteq G_{\beta}$. Положим

$$
S_{0}=\left\{a_{\alpha}: \alpha<\beta\right\} \cup\left\{g_{\alpha} a_{\alpha}: \alpha<\beta\right\}, \quad S_{1}=S \backslash S_{0} .
$$

Предположим, что $g S_{1} \cap F S \neq \varnothing$. Тогда найдутся такие ординалы $\alpha, \lambda, \alpha \geqslant \beta$, что

$$
\left\{g a_{\alpha}, g g_{\alpha} a_{\alpha}\right\} \cap\left\{F a_{\lambda}, F g_{\lambda} a_{\lambda}\right\} \neq \varnothing .
$$

Если $\lambda \neq \alpha$, то получаем противоречие с условием $(* *)$,поскольку $F^{-1} g \subseteq G_{\beta} \subseteq G_{\alpha}$. Если $\alpha=\lambda$, то вьполняется одно из следуюших условий:

$$
g \in F, \quad g \in F g_{\alpha}, \quad g \in F g_{\alpha}^{-1},
$$

каждое из которых противоречит выбору $g$. 
Итак, $g S_{1} \cap F S=\varnothing ;$ следовательно, $F S_{1} \subseteq F g^{-1}(G \backslash F S)$. Поскольку $\left|F S_{0}\right|<\gamma$, найдется такой элемент $h \in G$, что $h F S_{0} \cap F S_{0}=\varnothing$. Следовательно,

$$
\begin{gathered}
F S_{0} \subseteq h^{-1}\left(G \backslash F S_{0}\right) \subseteq h^{-1}(G \backslash F S) \cup h^{-1} F S_{1} \\
F S \subseteq h^{-1}(G \backslash F S) \cup h^{-1} F g^{-1}(G \backslash F S) \cup F g^{-1}(G \backslash F S)
\end{gathered}
$$

Положим $K=\left\{h^{-1}\right\} \cup h^{-1} F g^{-1} \cup F g^{-1} \cup\left\{g_{0}\right\}$. Тогда $G=K(G \backslash F S)$ и подмножество $G \backslash F S$ большое слева.

Предположим, что группа $G$ аменабельна. Используя условие (**), выберем индуктивно возрастающую последовательность $m_{1}, m_{2}, \ldots$ натуральных чисел так, чтобы $g_{n} S\left(m_{n}\right) \cap G_{n-1} S=\varnothing$, где $S(k)=\left\{a_{\alpha}: \alpha \geqslant k\right\} \cup\left\{g_{\alpha} a_{\alpha}: \alpha \geqslant k\right\}$. Ясно, что $\mu\left(g_{n} S\left(m_{n}\right)\right)$ $=\mu\left(S\left(m_{n}\right)\right)=\mu(S)$. По построению семейство $\left\{g_{n} S\left(m_{n}\right): 0<n<\omega\right\}$ дизъюнктно. Следовательно, $\mu(S)=0$.

Вопрос 1. Можно ли в любой бесконечной группе $G$ выбрать такое малое подмнохество $S$, что $G=S S^{-1}$ ?

ТЕОрема 3. Любую бесконечную әруппу $G$ мохнно разбить на счетное число подмнохеств $G=\bigcup_{n=1}^{\infty} S_{n}$ так, что $G=\operatorname{gp}\left(S_{n}\right)$ и каждое подмножество $S_{n}$ мало слева. Если группа $G$ аменабельна, то разбиение можно выбрать так, что $\mu\left(S_{n}\right)=0$ для любого подмножсества $S_{n}$ и любой банаховой меры $\mu$ на $G$.

ДокАЗАТЕльство. Вначале предположим, что группа $G$ порождается некоторым конечным подмножеством $F$, содержащим единицу е группы $G$. Выберем индуктивно последовательность $a_{1}, a_{2}, \ldots$ элементов групшы $G$ такую, что $a_{n} F \cap a_{m} F=\varnothing$ при $n \neq m$. Разобьем группу $G=\cup_{n=1}^{\infty} S_{n}$ на конечные подмножества так, что $a_{n} F_{n} \subseteq S_{n}$ и заметим, что каждое конечное подмножество бесконечной групшы мало.

Допустим, что группа $G$ не является конечно порожденной, $|G|=\gamma$. Построим индуктивно цепь $\left\{G_{\alpha}: \alpha<\gamma\right\}$ подгрупп групшы $G$ так, чтобы выполнялись следующие условия:

(i) $G_{0}=\{e\}, G=\cup\left\{G_{\alpha}: \alpha<\gamma\right\}$;

(ii) $G_{\alpha} \subset G_{\beta}$ для любых ординалов $\alpha<\beta<\gamma$;

(iii) $\cup\left\{G_{\alpha}: \alpha<\beta\right\}=G_{\beta}$ для любого предельного ординала $\beta$.

Для каждого ординала $\alpha<\gamma$ разложим подмножество $G_{\alpha+1} \backslash G_{\alpha}$ на правые смежные классы по подгрупше $G_{\alpha}$ и зафиксируем некоторое подмножество $X_{\alpha}$ представителей смежных классов. Таким образом, $G_{\alpha+1} \backslash G_{\alpha}=G_{\alpha} X_{\alpha}$.

Возьмем произвольньй элемент $g \in G, g \neq e$, и выберем наименьшую подгруппу $G_{\alpha}$, содержащую $g$. По условию (iii) $\alpha=\alpha_{1}+1$ для некоторого ординала $\alpha_{1}<\gamma$. Значит, $g \in G_{\alpha_{1}+1} \backslash G_{\alpha_{1}}$ и $g=g_{1} x_{\alpha_{1}}, g_{1} \in G_{\alpha_{1}}, x_{\alpha_{1}} \in X_{\alpha_{1}}$. Если $g_{1} \neq e$, выберем ординал $\alpha_{2}$, элементы $g_{2} \in G_{\alpha_{2}}, x_{\alpha_{2}} \in X_{\alpha_{2}}$ так, что $g_{1}=g_{2} x_{\alpha_{2}}$. Поскольку множество ординалов $<\gamma$ вполне упорядочено, через конечное число $s(g)$ шагов получим представление

$$
g=x_{\alpha_{s(g)}} x_{\alpha_{s(g)-1}} \ldots x_{\alpha_{2}} x_{\alpha_{1}}, \quad \alpha_{s(g)}<\cdots<\alpha_{1}, \quad x_{\alpha_{i}} \in X_{\alpha_{i}}
$$

Заметим, что такое представление однозначно и положим

$$
\nu_{1}(g)=\alpha_{1}, \quad \nu_{2}(g)=\alpha_{2}, \quad \ldots, \quad \nu_{s(g)}(g)=\alpha_{s(g)}, \quad \Gamma(y)=\left\{\nu_{1}(g), \ldots, \nu_{s(g)}(g)\right\}
$$


Для каждого натурального числа $n$ положим $D_{n}=\{g \in G: s(g)=n\}$. Покажем, что подмножество $G_{\alpha} D_{n}$ мало слева для всех $\alpha, n$. Возьмем любое конечное подмножество $F \subset G$ и выберем $\beta<\gamma$ так, чтобы $F \subset G_{\beta}$ и $G_{\alpha \subseteq} G_{\beta}$. Так как $F G_{\alpha} D_{n} \subseteq G_{\beta} D_{n}$, то достаточно показать, что подмножество $G \backslash G_{\beta} D_{n}$ большое слева. Зафиксируем элементы $a_{1}, a_{2}, \ldots, a_{n+1}$ группы $G$ такие, что

$$
a_{1} \in G_{\beta+1} \backslash G_{\beta}, \quad a_{2} \in G_{\beta+2} \backslash G_{\beta+1}, \ldots, \quad a_{n+1} \in G_{\beta+n+1} \backslash G_{\beta+n} .
$$

Возьмем любой элемент $g \in G_{\beta} D_{n}$ и положим $g=g_{0}$. Если $\beta+n \in \Gamma(g)$, то положим $\varepsilon_{0}=0 ;$ иначе, $\varepsilon_{0}=1$. Заметим, что $\beta+n \in \Gamma\left(a_{n+1}^{\varepsilon_{0}} g_{0}\right)$ и положим $g_{1}=a_{n+1}^{\varepsilon_{0}} g_{0}$. Если $\beta+n-1 \in \Gamma\left(g_{1}\right)$, то положим $\varepsilon_{1}=0$; иначе, $\varepsilon_{1}=1$. Заметим, что $\{\beta+n-1, \beta+n\} \subseteq$ $\Gamma\left(a_{n}^{\varepsilon_{1}} g_{1}\right)$, и положим $g_{2}=a_{n}^{\varepsilon_{1}} g_{1}$. После $(n+1)$-го шага получаем

$$
\{\beta, \beta+1, \ldots, \beta+n\} \subseteq \Gamma\left(a_{1}^{\varepsilon_{n}} a_{2}^{\varepsilon_{n-1}} \ldots a_{n+1}^{\varepsilon_{0}} g\right)
$$

Это значит, что $\left(a_{1}^{\varepsilon_{n}} a_{2}^{\varepsilon_{n-1}} \ldots a_{n+1}^{\varepsilon_{0}} g\right) \notin G_{\beta} D_{n}$. Обозначим $A=\left\{e, a_{1}, a_{2}, \ldots, a_{n+1}\right\}$, $K=A^{n+1}$. Мы доказали, что $K G_{\beta} D_{n} \cap G_{\beta} D_{n}=\varnothing$. Следовательно, $G=K^{-1}(G \backslash$ $\left.G_{\beta} D_{n}\right)$, т.е. подмножество $G_{\beta} D_{n}$ мало слева.

Положим $S_{1}=D_{1} \cup\{e\}, S_{2}=D_{2} \cup D_{3}, S_{3}=D_{4} \cup D_{5}, \ldots$ Ясно, что $G=\bigcup_{n=1}^{\infty} S_{n}$ и $S_{n} \cap S_{m}=\varnothing$ при $n \neq m$. Так как $D_{1} \subseteq \operatorname{gp}\left(S_{n}\right)$ и $\operatorname{gp}\left(D_{1}\right)=G$, то $G=\operatorname{gp}\left(S_{n}\right)$. Поскольку каждое подмножество $D_{n}$ мало слева, то и $S_{n}$ мало слева.

Предположим, что группа $G$ аменабельна. Если $G$ конечно порождена, то второе утверждение теоремы очевидно. Если $G$ не является конечно порожденной, то цеп подгрупп $\left\{G_{\alpha}: \alpha<\gamma\right\}$ можно выбрать так, чтобы она удовлетворяла дополнительному условию

(iv) $\left|G_{n+1}: G_{n}\right|>n$ для всех $n<\omega$.

Достаточно показать, что $\mu\left(D_{n}\right)=0$. Возьмем произвольное натуральное число $m$ и убедимся, что $\mu\left(D_{n}\right)<1 / m$. Положим $D_{n}(k)=\{g \in G:(n+1) m+k \notin \Gamma(g)\}$, $k \in\{0,1, \ldots, n\}$. Из определения подмножества $D_{n}$ следует, что $D_{n}=\bigcup_{k=0}^{n} D_{n}(k)$. Выберем произвольное подмножество $Y_{k}$ представителей левых смежных классов подгруппы $G_{(n+1) m+k+1}$ по подгруппе $G_{(n+1) m+k}$. Заметим, что семейство $\left\{y D_{n}(k)\right.$ : $\left.y \in Y_{k}\right\}$ дизъюнктно. Из условия (iv) следует, что

$$
\mu\left(D_{n}(k)\right)<\frac{1}{(n+1) m} .
$$

Значит, $\mu\left(D_{n}\right)<1 / m$.

Вопрос 2. Мохно ли любую бесконечную группу разбить на счетное число малых подмножеств, каждое из которых порождает группу?

Теорема 4. Пусть $H$ - группа, снабжсеная недискретной хаусдорфовой топологией $\tau$, в которой непрерывны все левые сдвиги $x \longmapsto h x, h \in H$. Тогда $H$ порождается подмножеством с пустой внутренностью. 
ДоКАЗАТЕЛЬСТВО. По лемме Цорна топологию $\tau$ можно усилить до максимальной недискретной левоинвариантной топологии $\tau^{\prime}$. Достаточно показать, что $\left(H, \tau^{\prime}\right)$ порождается некоторым дискретным подмножеством $D$. По определению топологии $\tau^{\prime}$ подмножество $D$ имеет пустую внутренность в $(H, \tau)$.

Пусть $\gamma$ - минимальная мощность непустьх открытых подмножеств группы $\left(H, \tau^{\prime}\right)$, $U$ - окрестность единицы $e$ в $\left(H, \tau^{\prime}\right)$ мощности $\gamma, G$ - подгруппа, порожденная подмножеством $U, H=G X$ - разложение групшы $H$ на правые смежные классы по подгруппе $G, e \in X$. Если $G$ порождается конечным подмножеством $F$, то $F X$ - дискретная система образующих $\left(H, \tau^{\prime}\right)$. Если $G$ не является конечно порожденной, то определим цепь подгрупп $\left\{G_{\alpha}: \alpha<\gamma\right\}$, удовлетворяющую условиям (i), (ii), (iii) предыдущей теоремы. По теореме 3.1 из [3] каждое подмножество $D_{n}$ дискретно и замкнуто. Следовательно, $D_{1} X$ - дискретная система образующих групшы $\left(H, \tau^{\prime}\right)$.

ВоПРос 3. Верно ли, что любая недискретная хаусдорфова левотопологическая группа порождается некоторым нигде не плотным подмножсеством?

Для счетных регулярных левотопологических групп ответ утвердительньй. Такая группа порождается даже некоторым дискретным замкнутым подмножеством. В [4] это доказано для топологических групп, однако некоторое усовершенствование доказательства позволяет распространить утверждение и на регулярные левотопологические группы.

По теореме 11.5 из [5], для любого конечного разбиения

$$
G=A_{1} \cup A_{2} \cup \cdots \cup A_{n}
$$

произвольной группы $G$ найдется такое подмножество $A_{i}$ разбиения, что справедливы следующие утверждения:

1) $G=K A_{i} A_{i}^{-1}$ для некоторого конечного подмножества $K \subseteq G$;

2) $\left(A_{i} A_{i}^{-1}\right)^{m}$ - подгруппа группы $G$ для некоторого натурального числа $m$;

3) $f\left(A_{i}\right) \cap A_{i} A_{i}^{-1} A_{i} A_{i}^{-1} \neq \varnothing$ для любого гомоморфизма $f: G \longrightarrow G$.

Следующая теорема, которой предшествуют три леммы, является уточнением этого результата.

Лемма 1. Пусть $F, A_{1}, A_{2}, \ldots, A_{n}-$ подмножсества группь $G$ такие, что

$$
G=F\left(A_{1} \cup A_{2} \cup \cdots \cup A_{n}\right)
$$

$u|F| \leqslant m, 1 \leqslant m<\omega$. Тогда найдутся индекс $i \in\{1, \ldots, n\}$, конечное подмножество $K \subseteq G$ такие, что

$$
G=K A_{i} A_{i}^{-1}, \quad|K| \leqslant f(n, m)
$$

әде функиия $f(n, m)$ определена рекуррентным соотношением

$$
f(1, m)=m, \quad f(n+1, m)=f\left(n, m+m^{2}\right) .
$$


ДокАЗАТЕЛЬСтво. Применим индукцию по $n$. Для $n=1$ имеем $G=F A_{1}$. Так как $G=F A_{1} g$ для любого элемента $g \in G$, то $G=F A_{1} A_{1}^{-1}$. Положим $K=F$.

Пусть $G=F\left(A_{1} \cup A_{2} \cup \cdots \cup A_{n+1}\right),|F| \leqslant m$. Рассмотрим два случая.

1. Существует элемент $g \in G$ такой, что $g A_{1} \subseteq F\left(A_{2} \cup A_{3} \cup \cdots \cup A_{n+1}\right)$. Тогда

$$
A_{1} \subseteq g^{-1} F\left(A_{2} \cup \cdots \cup A_{n+1}\right), \quad F A_{1} \subseteq F g^{-1} F\left(A_{2} \cup \cdots \cup A_{n+1}\right) .
$$

Следовательно,

$$
G=\left(F \cup F g^{-1} F\right)\left(A_{2} \cup \cdots \cup A_{n+1}\right) .
$$

Так как $\left|F \cup F g^{-1} F\right| \leqslant m+m^{2}$, по предположению индукции найдутся $i \in\{2, \ldots$, $n+1\}$ и конечное подмножество $K \subseteq G$ такие, что

$$
G=K A_{i} A_{i}^{-1}, \quad|K| \leqslant f\left(n, m+m^{2}\right)=f(n+1, m) .
$$

2. Для любого элемента $g \in G g A_{1}$ не содержится в подмножестве $F\left(A_{2} \cup A_{3} \cup \cdots\right.$ $\left.\cup A_{n+1}\right)$. Так как $G=F A_{1} \cup F\left(A_{2} \cup \cdots \cup A_{n+1}\right)$, то $g A_{1} \cap F A_{1} \neq \varnothing$ для любого элемента $g \in G$. Следовательно, $G=F A_{1} A_{1}^{-1}$.

Положим $K=F$ и заметим, что $|K| \leqslant m \leqslant f(n+1, m)$.

ЛЕмма 2. Функиия $f(n, m)$, определенная рекуррентным соотношением

$$
f(1, m)=m, \quad f(n+1, m)=f\left(n, m+m^{2}\right),
$$

удовлетворяет неравенству

$$
f(n, m) \leqslant 2^{2^{n-1}-1} m^{2^{n-1}} .
$$

ДоКАЗАТЕЛьСТво. Для $n=1$ справедливо равенство. Для $n>1$ имеем

$$
\begin{aligned}
f(n, m) & \leqslant f\left(n-1,2 m^{2}\right) \leqslant f\left(n-2,2^{1+2} m^{2^{2}}\right) \leqslant f\left(n-3,2^{1+2+2^{2}} m^{2^{3}}\right) \\
& \leqslant \cdots \leqslant f\left(1,2^{1+2+\cdots+2^{n-2}} m^{2^{n-1}}\right)=2^{2^{n-1}-1} m^{2^{n-1}} .
\end{aligned}
$$

Лемма 3. Пусть $G$ - әруппа с единицей $е, S \subseteq G, S=S^{-1}, e \in S$. Если $G=K S$, $|K|=k$, mo $S^{4^{k-1}}-$ noдгрynna гpynnы $G$.

ДокАЗАТЕЛЬСТво. Применим индукцию по $k$. Для $k=1$ утверждение очевидно. Пусть $G=a_{1} S \cup a_{2} S \cup \cdots \cup a_{k+1} S$. Если $a_{1} S^{2}=a_{1} S$, то $S$ - подгруппа. Иначе, найдется индекс $i \in\{2, \ldots, k+1\}$ такой, что $a_{1} S^{2} \cap a_{i} S \neq \varnothing$. Значит, $a_{1} \in a_{i} S^{3}$ и $a_{1} S \subseteq a_{i} S^{4}$. Следовательно, $G=a_{2} S^{4} \cup \cdots \cup a_{n+1} S^{4}$. По предположению индукции $\left(S^{4}\right)^{4^{k-1}}=S^{4^{k}}-$ подгруппа.

Tеорема 5. Eсли группа $G$ разбита на п частей $G=A_{1} \cup A_{2} \cup \cdots \cup A_{n}$, то найдутся такие подмножество разбиения $A_{i}$, натуральное число $k \leqslant 2^{2^{n-1}-1} u$ подмнохество $K \subseteq G$, что $G=K A_{i} A_{i}^{-1},|K|=k u\left(A_{i} A_{i}^{-1}\right)^{4^{k-1}}$ - подгруппа zpynnы $G$. 
ДокаЗАТЕЛЬСтво. Применяем лемму 1 с подмножеством $F=\{e\}$, а затем леммы 2,3 .

Теорема 6. Если аменабельная группа $G$ разбита на п частей $G=A_{1} \cup A_{2} \cup \ldots$ $\cup A_{n}$, то найдутся такие подмножество разбиения $A_{i}$ и подмножество $K \subseteq G$, $|K| \leqslant n$, что $G=K A_{i} A_{i}^{-1} u\left(A_{i} A_{i}^{-1}\right)^{4^{n-1}}-$ nодгруnпа груnпьь $G$.

ДокАЗАтЕЛьство. Пусть $\mu$ - фиксированная банахова мера на $G$. Выберем подмножество $A_{i}$ максимальной меры $\mu\left(A_{i}\right) \geqslant 1 / n$. Выберем максимальное дизъюнктное семейство подмножеств $\left\{a_{k} A_{i}: k \leqslant n\right\}$ групшы $G$ и положим $K=\left\{a_{1}, \ldots, a_{k}\right\}$. Возьмем произвольный элемент $g \in G$. Тогда $g A_{i} \cap a_{k} A_{i} \neq \varnothing$ для некоторого $k \leqslant n$. Следовательно, $g \in a_{k} A_{i} A_{i}^{-1}$ и $G=K A_{i} A_{i}^{-1}$. Далее применяем лемму 3.

Вопрос 4 [6, задача 13.44]. Пусть группа $G$ разбита на п частей $G=A_{1} \cup A_{2} \cup \cdots$ $\cup A_{n}$. Верно ли, что найдутся такие подмножество $K \subseteq G,|K|=n$, и подмножество разбиения $A_{i}$, что $G=K A_{i} A_{i}^{-1}$ ?

Для $n \leqslant 2$ это так по теореме 5 . Для аменабельной групшы $G$ и произвольного $n$ ответ также положительный по теореме 6 .

\section{СПИСОК ЦИТИРОВАННОЙ ЛИТЕРАТУРЫ}

[1] Bella A., Malykhin V. I. On certain subsets of a group // Questions Answers Gen. Topology. 1999. V. 17. № 2. P. 183-197.

[2] Malykhin V. I., Moresco R. Small generated groups // Questions Answers Gen. Topology. 2001. V. 19. P. 1-7.

[3] Протасов И. В. Максимальные топологии на группах // Сиб. матем. ж. 1998. Т. 39. №6. C. $1368-1381$.

[4] Comfort W. W., Morris S. A., Robbie D., Svetlichny S., Tkachenko M. Suitable sets for topological groups // Topology Appl. 1998. V. 86. № 1. P. 25-46.

[5] Protasov I. Combinatorics of Numbers. Math. Stud. Monograph Ser. V. 2: VNTL Publishers, 1997.

[6] Нерешенные вопросы теории групп. Коуровская тетрадь. 13-е изд. Новосибирск, 1995.

Киевский национальный университет им. Т. Шевченка

Поступило

E-mail: kseniya@profit.net.ua

21.11.2001

Исправленный вариант

01.07.2002 